\title{
Global solution to the Cauchy problem of the nonlinear double dispersive wave equation with strong damping
}

\author{
C. Song and Z. Yang \\ Communicated by Y. Charles Li, received November 2, 2008.
}

\begin{abstract}
This paper concerns with the global classical solution to the Cauchy problem of the nonlinear double dispersive wave equation with strong damping

$$
u_{t t}-\Delta u-\Delta u_{t t}+\Delta^{2} u-c \Delta u_{t}=d \sum_{i=1}^{N} \frac{\partial}{\partial x_{i}} \sigma_{i}\left(u_{x_{i}}\right),
$$

where $c$ and $d$ are positive constants. By the contraction mapping principle and priori estimates, we prove that the Cauchy problem admits a unique global classical solution, and by the concavity method, we give the sufficient conditions on the blowup of the global solution of the Cauchy problem. Finally, as an application, an example is also given.
\end{abstract}

\section{CONTEnTS}

1. Introduction 367

2. Main results $\quad 369$

3. Existence and uniqueness of the local solution 371

4. Existence and uniqueness of the global classical solution 376

5. Blow up of solutions for the Cauchy problem (1.8)-(1.9) 378

References

\section{Introduction}

In 1872, J. Boussinesq [1] derived model equation

$$
u_{t t}-u_{x x}+\alpha u_{x x x x}=\beta\left(u^{2}\right)_{x x}
$$

1991 Mathematics Subject Classification. 35L05, 35L35, 35L75.

Key words and phrases. Cauchy problem, double dispersive wave equation, global solution, strong damping.

This work is supported by Natural Science Foundation of China (No. 10971199), Natural Science Foundations of Henan Province (No. 092300410067, No. 082300410080) and Natural Science Foundation of Department of Education of Henan Province of China (No. 2008B110024). 
to describe the propagation of long waves on the surface of shallow water, where $u(x, t)$ is an elevation of the free surface of fluid, subscripts denote partial derivatives, and $\alpha>0$ and $\beta$ are real numbers depending on the depth of fluid and the characteristic speed of long waves. For the equation (1.1) and its various generalized forms, there have been a lot of impressive works from both local and global well-posedness to the blowup of solutions [see, for example, 3-4, 6-7, 10, 13-14]. Since equation (1.1) is a nonlinear evolution equation describing wave motion in a medium with dissipation, it is interesting to take into account the effects of viscosity. V. Varlamov [2] studied the damped Boussinesq equation

$$
u_{t t}-u_{x x}+\alpha u_{x x x x}-2 b u_{x x t}=\beta\left(u^{2}\right)_{x x}
$$

and proved the local in time existence and uniqueness of smooth solutions of the Cauchy problem, where $2 b u_{x x t}$ represents the internal strong damping. The equation of (1.2)-type with weak damping $u_{t}$ and a linear feed-back term $(u-[u])$ on a periodic domain has been examined in [5] from the point of view of establishing the global well-posedness.

In the classical paper [1], Boussinesq also derived an approximate equation from the Euler equation for surface wave in irrotational motion

$$
u_{t t}-u_{x x}-\frac{\varepsilon}{2} u_{x x t t}+\frac{\varepsilon}{6} u_{x x x x}-3 \varepsilon u_{x} u_{x x}=0,
$$

where $\varepsilon>0$ is parameter. By virtue of a priori estimate and the successive approximation, T. Kano and T. Nishida [8] investigated the global existence of the Cauchy problem for the revised version of equation (1.3)

$$
u_{t t}-u_{x x}-\frac{\varepsilon}{2} u_{x x t t}+\frac{\varepsilon}{6} u_{x x x x}+2 \varepsilon u_{x} u_{x t}+\varepsilon u_{t} u_{x x}=0 .
$$

Since equation (1.3) includes two dispersive terms, we call it double dispersive wave equation. Its generalized form of (1.3) is as follows

$$
u_{t t}-u_{x x}-a u_{x x t t}+b u_{x x x x}=d \sigma\left(u_{x}\right)_{x},
$$

where $a, b$ and $d$ are positive constants, $\sigma$ is a given nonlinear function. The equations (1.3) and (1.5) take into account the effects of dispersion and nonlinearity, but in real processes, if we also consider the effects of the strong internal damping $[2,15]$, then the double dispersion wave equation with strong damping is as follows

$$
u_{t t}-u_{x x}-a u_{x x t t}+b u_{x x x x}-c u_{x x t}=d \sigma\left(u_{x}\right)_{x} .
$$

The $N$ dimension model of (1.6) is

$$
u_{t t}-\triangle u-a \triangle u_{t t}+b \triangle^{2} u-c \triangle u_{t}=d \sum_{i=1}^{N} \frac{\partial}{\partial x_{i}} \sigma_{i}\left(u_{x}\right) .
$$

We can assume that the dispersion coefficient $a=b=1$ by the scaling transformation $u(x, t) \longrightarrow u\left(\sqrt{a} x, \frac{a}{\sqrt{b}} t\right)$. Also, we can assume that $\sigma_{i}(0)=0$, otherwise, we can replace $\sigma_{i}(s)$ by $\sigma_{i}(s)-\sigma_{i}(0)$. Thus, without loss of generality, we study the following Cauchy problem

$$
\begin{aligned}
& u_{t t}-\triangle u-\triangle u_{t t}+\triangle^{2} u-c \triangle u_{t}=d \sum_{i=1}^{N} \frac{\partial}{\partial x_{i}} \sigma_{i}\left(u_{x_{i}}\right), x \in \mathbf{R}^{N}, t>0, \\
& u(x, 0)=u_{0}(x), \quad u_{t}(x, 0)=u_{1}(x), x \in \mathbf{R}^{N}
\end{aligned}
$$


where $c$ and $d$ are positive constants, $u_{0}(x)$ and $u_{1}(x)$ are given initial functions and $\sigma_{i}(s)(i=1, \cdots, N)$ are given nonlinear functions.

One of the methods of studying Cauchy problems for high-order nonlinear evolution equations is the inverse scattering transform [11]. However, this technique does not work for a wide class of dissipative equation which are not completely integrable. Another approach has been proposed by Naumkin and Shishmarev [12], By means of using both the spectral and perturbation theories they have succeeded in constructing the exact solutions of the Cauchy problem. In 1996, Varlamov [2] developed the method to investigate that of the damped Boussinesq equation and proved the local in time existence and uniqueness of smooth solution. Since the equation (1.8) includes the dispersive term $\triangle u_{t t}$, this approach can not be employed. In the present paper, we first reduce the Cauchy problem (1.8)-(1.9) to an equivalent Cauchy problem for a differential integral equation by the fundamental solution of a second-order partial differential equation, and then prove the existence and uniqueness of the local solution of the equivalent Cauchy problem by the contraction mapping principle, namely, the Cauchy problem (1.8)-(1.9) admits a unique local solution. Finally we prove the existence and uniqueness of global solution of the Cauchy problem (1.8)-(1.9) by priori estimates.

The paper is organized as follows. In Section 2, some notations, Lemmas and the main results are stated. The existence and uniqueness of local solution of the problem (1.8)-(1.9) are studied by the contraction principle in Section 3. The existence and uniqueness of global solution of the problem (1.8)-(1.9) are investigated by the priori estimates in Section 4. The sufficient conditions on the blowup of the global solution of the problem (1.8)-(1.9) is discussed by the concavity method in Section 5 , and an application is given in the last section.

\section{Main results}

We use the following abbreviations: $\|\cdot\|_{p}=\|\cdot\|_{L_{p}\left(R^{N}\right)}(1 \leq p \leq+\infty)$ denotes usual $L_{p}$ norm, $\|\cdot\|=\|\cdot\|_{L_{2}\left(R^{N}\right)},(\cdot, \cdot)$ denotes the $L_{2}$-inner product, and equip the Sobolev space $H^{r}\left(R^{N}\right)$ with the norm

$$
\|f\|_{H^{r}}=\left\|(I-\triangle)^{r / 2} f\right\|
$$

for each real number $r$, where $I$ denotes an unitary operator. Throughout this paper, $u * v$ is the convolution defined by

$$
u * v(x)=\int_{\mathbf{R}} u(y) v(x-y) d y .
$$

We need the following lemmas,

Lemma 2.1 ${ }^{[9]}$. Assume that $g(u) \in C^{m}(\mathbf{R})(m \geq 1)$ and $g(0)=0$. Then for $u \in H^{m} \cap L_{\infty}$, we have

$$
\|g(u)\|_{H^{m}} \leq M_{0}\left(\|u\|_{\infty}\right)\|u\|_{H^{m}},
$$

where $M_{0}\left(\|u\|_{\infty}\right)$ is a constant dependent on $\|u\|_{\infty}$.

Lemma 2.2 ${ }^{[9]}$ (Sobolev multiplier lemma). Assume that $m_{1}, m_{2} \geq m>\frac{N}{2}$. Then for $u \in H^{m_{1}}, v \in H^{m_{2}}$, we have

$$
\|u v\|_{H^{m}} \leq C\|u\|_{H^{m_{1}}}\|v\|_{H^{m_{2}}},
$$

where $C$ is a positive constant independent of $u$ and $v$. 
Lemma 2.3 ${ }^{[18]}$ (Sobolev imbedding theorem). For $m>k+\frac{N}{2}, k \geq 0$ is an integer, then

$$
H^{m} \subset C^{k}\left(R^{N}\right) \cap L_{\infty}
$$

Lemma 2.4 ${ }^{[16]}$. Assume that $F \in C^{2}(\mathbf{R}), F(t) \geq 0, t \geq 0$ and $F(0)>0$, $F^{\prime}(0)>0$, and

$$
F^{\prime \prime}(t) F(t)-(\gamma+1)\left[F^{\prime}(t)\right]^{2} \geq 0,
$$

where $\gamma>0$ is a real number. Then there is a $t_{1} \leq t_{2}=F(0) / \gamma F^{\prime}(0)$ such that $F(t) \rightarrow \infty$ as $t \rightarrow t_{1}$.

In the following, we state the main results.

Theorem 2.1. Assume that $m>1+\frac{N}{2}, u_{0} \in H^{m+3}, u_{1} \in H^{m+2}, \sigma_{i}(s) \in$ $C^{m+2}, i=1, \cdots, N$. Then the Cauchy problem (1.8)-(1.9) admits a unique local solution $u(x, t)$ with

$$
u \in C\left(\left[0, T^{0}\right) ; H^{m+3}\right) \cap C^{1}\left(\left[0, T^{0}\right) ; H^{m+2}\right) \cap C^{2}\left(\left[0, T^{0}\right) ; H^{m+1}\right),
$$

where $\left[0, T^{0}\right)$ is the maximal existence interval, $T^{0}>0$. Moreover, if

$$
\sup _{0 \leq t<T^{0}}\left[\|u(t)\|_{H^{m+3}}^{2}+\left\|u_{t}(t)\right\|_{H^{m+2}}^{2}+\left\|u_{t t}(t)\right\|_{H^{m+1}}^{2}\right]<+\infty,
$$

then $T^{0}=+\infty$.

Theorem 2.2. Assume that $m>1+\frac{N}{2}, u_{0} \in H^{m+3}, u_{1} \in H^{m+2}, \sigma_{i}(s) \in$ $C^{m+2}, \sigma^{\prime}(s)$ is bounded below, namely, there exists a constant $C_{i}$ such that

$$
\sigma_{i}^{\prime}(s) \geq C_{i}, s \in \mathbf{R}, i=1, \cdots, N
$$

Then the Cauchy problem (1.8)-(1.9) admits a unique global classical solution $u(x, t)$ with

$$
u \in C\left([0, \infty) ; C^{4}\right) \cap C^{1}\left([0, \infty) ; C^{3}\right) \cap C^{2}\left([0, \infty) ; C^{2}\right) .
$$

Theorem 2.3. Assume that

(1) The assumptions of Theorem 2.1 hold, $\Gamma_{i}\left(u_{0 x_{i}}\right) \in L_{1}\left(R^{N}\right)$, where $\Gamma_{i}(s)=$ $\int_{0}^{s} \sigma_{i}(\tau) d \tau, i=1, \cdots, N$.

(2) There exists a constant $\gamma>0$ such that

$$
d \sigma_{i}(s) s+c^{2} s^{2} \leq(3+4 \gamma) d \Gamma_{i}(s), \quad s \in \mathbf{R}, i=1, \cdots, N .
$$

(3) One of the following conditions holds

(i) $E(0)=\left\|u_{1}\right\|^{2}+\left\|\nabla u_{0}\right\|^{2}+\left\|\nabla u_{1}\right\|^{2}+\left\|\triangle u_{0}\right\|^{2}+\sum_{i=1}^{N} \int_{R^{N}} \Gamma_{i}\left(u_{0 x_{i}}\right) d x<0$;

(ii) $E(0)=0$ and $\left(u_{0}, u_{1}\right)+\left(\nabla u_{0}, \nabla u_{1}\right)>0$;

(iii) $E(0)>0$ and $\left(u_{0}, u_{1}\right)+\left(\nabla u_{0}, \nabla u_{1}\right)>\sqrt{\frac{2(1+\gamma)}{1+2 \gamma} E(0)\left(\left\|u_{0}\right\|^{2}+\left\|\nabla u_{0}\right\|^{2}\right)}$.

Then the solution $u=u(x, t)$ of the Cauchy problem (1.8)-(1.9) must blow up in a finite time.

Remark. The conditions of Theorem 2.3 can be reduced if we only consider the nonexistence of global solutions of the Cauchy problem (1.8)-(1.9). We can assume that $u_{0} \in H^{2}, u_{1} \in H^{1}, \Gamma_{i}\left(u_{0 x_{i}}\right) \in L_{1}\left(R^{N}\right)$, conditions (2) and (3) of Theorem 2.3 hold. Then the global solutions of Cauchy problem (1.8)-(1.9) cease to exist in a finite time. 


\section{Existence and uniqueness of the local solution}

Let $P(x)$ be a fundamental solution of the partial differential equation

$$
y(x)-\triangle y(x)=0 .
$$

By virtue of Fourier transform, we get

$$
P(x)=\frac{1}{(4 \pi)^{N / 2}} \int_{0}^{\infty} e^{-\left(\frac{|x|^{2}}{4 \tau}+\tau\right)} \tau^{-N / 2} d \tau, \quad x \in R^{N},
$$

and we can prove that $P(x)$ satisfies the following properties:

Lemma 3.1. (i) $P(x)$ is defined and continuous on $R^{N}, P(x) \in L_{p}\left(R^{N}\right)$ and $\|P\|_{1}=1$, where $1 \leq p \leq \infty$ if $N=1,1 \leq p<\infty$ if $N=2,1 \leq p<\frac{N}{N-2}$ if $N \geq 3$.

(ii) $P(x)$ satisfies the equation

$$
P(x)-\triangle P(x)=\delta(x),
$$

where $\delta(x)$ is the Dirac function;

(iii) $\|P * u\|_{H^{m}}=\|u\|_{H^{m-2}}$.

Proof. (i). By virtue of the Minkowski inequality we have

$$
\begin{aligned}
\|P\|_{p} & \leq \frac{1}{(4 \pi)^{N / 2}} \int_{0}^{\infty} e^{-\tau} \tau^{-N / 2}\left(\int_{R^{N}} e^{-\frac{p}{4 \tau}|x|^{2}} d x\right)^{1 / p} d \tau \\
& =\frac{1}{(4 \pi)^{N / 2}} \int_{0}^{\infty}(4 \tau \pi / p)^{N / 2 p} e^{-\tau} \tau^{-N / 2} d \tau \\
& =(4 \pi)^{\frac{N(1-p)}{2 p}} \int_{0}^{\infty} e^{-\tau} \tau^{\left(\frac{N}{2 p}-\frac{N}{2}+1\right)-1} d \tau \\
& =(4 \pi)^{\frac{N(1-p)}{2 p}} \Gamma\left(\frac{N}{2 p}-\frac{N}{2}+1\right),
\end{aligned}
$$

where $\Gamma(\cdot)$ denotes the Gamma function. By the definition of Gamma function we know that (i) holds.

(ii). The proof of (ii) can be seen in [19].

(iii). Since

$$
\begin{aligned}
& u=P *(I-\triangle) u=(I-\triangle)(P * u), P * u=(I-\triangle)^{-1} u \\
& \begin{aligned}
\|P * u\|_{H^{m}} & =\left\|(I-\triangle)^{m / 2}(P * u)\right\|=\left\|(I-\triangle)^{m / 2}(I-\triangle)^{-1} u\right\| \\
& =\|u\|_{H^{m-2}} .
\end{aligned}
\end{aligned}
$$

(iii) is proved.

Observe that equation (1.8) can be written as

$$
(I-\triangle)\left(u_{t t}-\triangle u+c u_{t}\right)=d \sum_{i=1}^{N} \frac{\partial}{\partial x_{i}} \sigma_{i}\left(u_{x_{i}}\right)+c u_{t}
$$

Set

$$
w=(I-\triangle) u
$$

therefore, equation (3.1) is equivalent to the following differential-integral equation

$$
w_{t t}-\triangle w=d \sum_{i=1}^{N} \frac{\partial}{\partial x_{i}} \sigma_{i}\left(P * w_{x_{i}}\right)+c P * w_{t}-c w_{t} .
$$


And hence the Cauchy problem (1.8)-(1.9) is equivalent to the Cauchy problem for the equation (3.2) with initial data

$(3.3) w(x, 0)=(I-\triangle) u_{0}(x)=w_{0}(x), \quad w_{t}(x, 0)=(I-\triangle) u_{1}(x)=w_{1}(x)$.

In the following, we prove that the Cauchy problem (3.2)-(3.3) admits a unique local solution by the contraction mapping principle, for this goal, we first consider the Cauchy problem for linear wave equation

$$
w_{t t}-\triangle w=f(x, t), \quad x \in R^{N}, t>0
$$

with initial data (3.3), we quote the following lemma.

Lemma 3.2 ${ }^{[17]}$. Assume that $w_{0} \in H^{m+1}, w_{1} \in H^{m}, m \geq 0$ is an integer; for any $T>0, f \in L_{2}\left(0, T ; H^{m}\right) \cap C\left([0, T] ; H^{m-1}\right)$, then the Cauchy problem (3.4)-(3.3) admits a unique solution satisfying

$$
\begin{aligned}
& \|w(t)\|_{H^{m+1}}^{2}+\left\|w_{t}(t)\right\|_{H^{m}}^{2}+\left\|w_{t t}(t)\right\|_{H^{m-1}}^{2} \\
\leq \quad & 6 e^{3 T}\left(\left\|w_{0}\right\|_{H^{m+1}}^{2}+\left\|w_{1}\right\|_{H^{m}}^{2}+\int_{0}^{t}\|f(\tau)\|_{H^{m}}^{2} d \tau\right) \\
& +2 \sup _{0 \leq t \leq T}\|f(t)\|_{H^{m-1}}^{2}, \quad 0 \leq t \leq T,
\end{aligned}
$$

In order to prove Theorem 2.1, we first prove the following Lemma.

Lemma 3.3. Assume that $m>1+\frac{N}{2}, w_{0} \in H^{m+1}, w_{1} \in H^{m}, \sigma_{i}(s) \in C^{m+2}$, then the Cauchy problem (3.2)-(3.3) admits a unique local solution $w(x, t)$ with

$$
w \in C\left(\left[0, T^{0}\right) ; H^{m+1}\right) \cap C^{1}\left(\left[0, T^{0}\right) ; H^{m}\right) \cap C^{2}\left(\left[0, T^{0}\right) ; H^{m-1}\right),
$$

where $\left[0, T^{0}\right)$ is the maximal existence interval, $T^{0}>0$. Moreover, if

$$
\sup _{0 \leq t<T^{0}}\left[\|w(t)\|_{H^{m+1}}^{2}+\left\|w_{t}(t)\right\|_{H^{m}}^{2}+\left\|w_{t t}(t)\right\|_{H^{m-1}}^{2}\right]<+\infty,
$$

then $T^{0}=+\infty$.

proof. Construct the space

$$
\begin{aligned}
B(K, T)= & \left\{v \mid v \in C\left([0, T] ; H^{m+1}\right) \cap C^{1}\left([0, T] ; H^{m}\right) \cap C^{2}\left([0, T] ; H^{m-1}\right),\right. \\
& v(x, 0)=w_{0}, v_{t}(x, 0)=w_{1}, \\
& \left.\sup _{0 \leq t \leq T}\left[\|v(t)\|_{H^{m+1}}^{2}+\left\|v_{t}(t)\right\|_{H^{m}}^{2}+\left\|v_{t t}(t)\right\|_{H^{m-1}}^{2}\right] \leq K^{2}\right\},
\end{aligned}
$$

where $K, T>0, w_{0} \in H^{m+1}, w_{1} \in H^{m}$. Define the norm as follows

$$
\|v\|_{B}=\sup _{0 \leq t \leq T}\left[\|v(t)\|_{H^{m+1}}^{2}+\left\|v_{t}(t)\right\|_{H^{m}}^{2}+\left\|v_{t t}(t)\right\|_{H^{m-1}}^{2}\right]^{1 / 2} .
$$

Obviously, $B(K, T)$ is a nonempty complete metric space. For $v \in B(K, T)$, we consider the Cauchy problem for the linear equation

$$
w_{t t}-\triangle w=h(x, t)
$$

with the initial data (3.3), where

$$
h(x, t)=d \sum_{i=1}^{N} \frac{\partial}{\partial x_{i}} \sigma_{i}\left(P * v_{x_{i}}\right)+c P * v_{t}-c v_{t} .
$$

Let $A$ denote the mapping which carries $v$ into the unique solution to the linear problem (3.9)-(3.3). Now we employ the contraction mapping principle to show $A$ has a unique fixed point in $B(K, T)$. 
In the following, we divide the proofs into three steps.

Step 1. We prove that A maps $B(K, T)$ into $B(K, T)$.

For any $v \in B(K, T)$, by the Sobolev embedding theorem and Lemma 3.1 we have

$$
P * v_{x_{i}} \in C\left([0, T] \times R^{N}\right), \quad i=1, \cdots, N
$$

And (3.7) implies

$$
\sup _{(x, t) \in R^{N} \times[0, T]}\left|P * v_{x_{i}}\right| \leq K, \quad v \in B(K, T), i=1, \cdots, N .
$$

In the following we show that the linear problem (3.9)-(3.3) satisfies the conditions of Lemma 3.2. By Lemma 2.1 and Lemma 2.2 we obtain

$$
\begin{aligned}
& \int_{0}^{t}\|h(\tau)\|_{H^{m}}^{2} d \tau \\
\leq & 3 \int_{0}^{t}\left[d^{2} \sum_{i=1}^{N}\left\|\sigma_{i}\left(P * v_{x_{i}}\right)\right\|_{H^{m+1}}^{2}\right. \\
& \left.+c^{2}\left(\left\|P * v_{t}(\tau)\right\|_{H^{m}}^{2}+\left\|v_{t}(\tau)\right\|_{H^{m}}^{2}\right)\right] d \tau \\
\leq \quad & 3 \int_{0}^{t}\left[d^{2} M_{0}(K)^{2}\|P * \nabla v(\tau)\|_{H^{m+1}}^{2}\right. \\
& \left.+c^{2}\left(\left\|P * v_{t}(\tau)\right\|_{H^{m}}^{2}+\left\|v_{t}(\tau)\right\|_{H^{m}}^{2}\right)\right] d \tau \\
\leq \quad & 3\left[d^{2} M_{0}(K)^{2}+2 c^{2}\right] K^{2} T . \\
& \left\|h_{t}(t)\right\|_{H^{m-1}} \\
\leq \quad & d \sum_{i=1}^{N}\left\|\sigma_{i}^{\prime}\left(P * v_{x_{i}}\right)\left(P * v_{x_{i} t}\right)\right\|_{H^{m}} \\
& +c\left(\left\|P * v_{t t}(t)\right\|_{H^{m-1}}+\left\|v_{t t}(t)\right\|_{H^{m-1}}\right) \\
\leq \quad & d C M_{0}(K) K^{2}+2 c K,
\end{aligned}
$$

and hence it follows from (3.12) that

$$
\begin{aligned}
\sup _{0 \leq t \leq T}\|h(t)\|_{H^{m-1}} & =\sup _{0 \leq t \leq T}\left\|h(0)+\int_{0}^{t} h_{t}(\tau) d \tau\right\|_{H^{m-1}} \\
\leq & \sup _{0 \leq t \leq T}\left[\|h(0)\|_{H^{m-1}}+\int_{0}^{t}\left\|h_{t}(\tau)\right\|_{H^{m-1}} d \tau\right] \\
\leq & d \sum_{i=1}^{N}\left\|\sigma_{i}\left(P * w_{0 x_{i}}\right)\right\|_{H^{m}}+c\left(\left\|P * w_{1}\right\|_{H^{m-1}}+\left\|w_{1}\right\|_{H^{m-1}}\right) \\
& +\left[d C M_{0}(K) K^{2}+2 c K\right] T .
\end{aligned}
$$

From (3.11) and (3.13) we know that

$$
h \in L_{2}\left([0, T] ; H^{m}\right) \cap C\left([0, T] ; H^{m-1}\right),
$$


therefore, by Lemma 3.2 we have

$$
\begin{aligned}
& \|w(t)\|_{H^{m+1}}^{2}+\left\|w_{t}(t)\right\|_{H^{m}}^{2}+\left\|w_{t t}(t)\right\|_{H^{m-1}}^{2} \\
\leq \quad & 6 e^{3 T}\left(\left\|w_{0}\right\|_{H^{m+1}}^{2}+\left\|w_{1}\right\|_{H^{m}}^{2}\right)+18 e^{3 T}\left[d^{2} M_{0}(K)^{2}+2 c^{2}\right] K^{2} T \\
& +4\left[d \sum_{i=1}^{N}\left\|\sigma_{i}\left(P * w_{0 x_{i}}\right)\right\|_{H^{m}}+c\left(\left\|P * w_{1}\right\|_{H^{m-1}}+\left\|w_{1}\right\|_{H^{m-1}}\right)\right]^{2} \\
& +4\left[d C M_{0}(K) K^{2}+2 K\right]^{2} T^{2} .
\end{aligned}
$$

Take $K$ big enough such that

$$
K^{2} \geq 12 e^{3}\left(\left\|w_{0}\right\|_{H^{m+1}}^{2}+\left\|w_{1}\right\|_{H^{m}}^{2}\right)
$$

$$
+8\left[d \sum_{i=1}^{N}\left\|\sigma\left(P * w_{0 x_{i}}\right)\right\|_{H^{m}}+c\left(\left\|P * w_{1}\right\|_{H^{m-1}}+\left\|w_{1}\right\|_{H^{m-1}}\right)\right]^{2},
$$

for the above $K$, we choose $T$ such that

$$
T \leq \min \left\{1,\left[36 e^{3}\left(d^{2} M_{0}(K)^{2}+2 c^{2}\right)+8\left(d C M_{0}(K) K+2\right)\right]^{-1}\right\} .
$$

Hence by (3.14), (3.15) and (3.16) we deduce that $A$ maps $B(K, T)$ into $B(K, T)$.

Step 2. We prove that the mapping $A: B(K, T) \longrightarrow B(K, T)$ is strictly contractive.

In fact, for any $v, \bar{v} \in B(K, T)$, let $w=A v, \bar{w}=A \bar{v}, W=w-\bar{w}, V=v-\bar{v}$, so $W$ satisfies the following Cauchy problem

$$
\begin{aligned}
& W_{t t}-\triangle W=G(x, t),(x, t) \in R^{N} \times[0, T], \\
& W(x, 0)=0, W_{t}(x, 0)=0, x \in R^{N},
\end{aligned}
$$

where

$$
G(x, t)=d \sum_{i=1}^{N} \frac{\partial}{\partial x_{i}}\left[\sigma_{i}\left(P * v_{x_{i}}\right)-\sigma_{i}\left(P * \bar{v}_{x_{i}}\right)\right]+c P * V_{t}-c V_{t}
$$

From Lemma 2.1, Lemma 2.2 and (3.19) we get

$$
\begin{aligned}
& \int_{0}^{t}\|G(\tau)\|_{H^{m}}^{2} d \tau \\
\leq & 3 \int_{0}^{t}\left[d^{2} \sum_{i=1}^{N}\left\|\sigma_{i}^{\prime}\left(P *\left(\theta_{1} v_{x_{i}}+\left(1-\theta_{1}\right) \bar{v}_{x_{i}}\right)\right)\left(P * V_{x_{i}}\right)\right\|_{H^{m+1}}^{2}\right. \\
& \left.+c^{2}\left(\left\|P * V_{t}(\tau)\right\|_{H^{m}}^{2}+\left\|V_{t}(\tau)\right\|_{H^{m}}^{2}\right)\right] d \tau \\
\leq & 3 \int_{0}^{t}\left[C^{2} d^{2} M_{0}(N)^{2} \| P *\left(\theta_{1} \nabla v\right.\right. \\
& \left.+\left(1-\theta_{1}\right) \nabla \bar{v}\right)(\tau)\left\|_{H^{m+1}}^{2}\right\| P * \nabla V(\tau) \|_{H^{m+1}}^{2} \\
& \left.+c^{2}\left(\left\|P * V_{t}(\tau)\right\|_{H^{m}}^{2}+\left\|V_{t}(\tau)\right\|_{H^{m}}^{2}\right)\right] d \tau \\
\leq \quad & 3 T\left[C^{2} d^{2} M_{0}(K)^{2} K^{2}+2 c^{2}\right] \sup _{0 \leq t \leq T}\left[\|V(t)\|_{H^{m+1}}^{2}+\left\|V_{t}(t)\right\|_{H^{m}}^{2}\right]
\end{aligned}
$$


where $0<\theta_{1}<1$ is a constant. Noting that the fact $G(x, 0)=0$ we have

$$
\begin{aligned}
& \sup _{0 \leq t \leq T}\|G(t)\|_{H^{m-1}}^{2}=\sup _{0 \leq t \leq T}\left\|G(0)+\int_{0}^{t} G_{t}(\tau) d \tau\right\|_{H^{m-1}}^{2} \\
\leq & \sup _{0 \leq t \leq T}\left(\int _ { 0 } ^ { t } \left\{d \sum _ { i = 1 } ^ { N } \left[\| \int_{0}^{1} \sigma_{i x_{i}}^{\prime \prime}\left(P * \left(\theta v_{x_{i}}\right.\right.\right.\right.\right. \\
& \left.\left.+(1-\theta) \bar{v}_{x_{i}}\right)\right) d \theta \cdot\left(P * V_{x_{i}}\right)\left(P * v_{x_{i}}\right) \|_{H^{m-1}} \\
& \left.+\left\|\sigma_{i x_{i}}^{\prime}\left(P * \bar{v}_{x_{i}}\right)\left(P * V_{x_{i} t}\right)\right\|_{H^{m-1}}\right] \\
& \left.\left.+c\left(\left\|P * V_{t t}(\tau)\right\|_{H^{m-1}}+\left\|V_{t t}(\tau)\right\|_{H^{m-1}}\right)\right\} d \tau\right)^{2} \\
\leq \quad & 3 T^{2}\left[C^{2} d^{2} M_{0}(K)^{2}\left(K^{4}+K^{2}\right) \sup _{0 \leq t \leq T}\left(\|V(t)\|_{H^{m+1}}^{2}+\left\|V_{t}(t)\right\|_{H^{m}}^{2}\right)\right. \\
& \left.+4 c^{2} \sup _{0 \leq t \leq T}\left\|V_{t t}(t)\right\|_{H^{m-1}}^{2}\right] .
\end{aligned}
$$

Therefore, by Lemma 3.1 we have

$$
\begin{aligned}
& \|W(t)\|_{H^{m+1}}^{2}+\left\|W_{t}(t)\right\|_{H^{m}}^{2}+\left\|W_{t t}(t)\right\|_{H^{m-1}}^{2} \\
\leq \quad & 18 T e^{3 T}\left[C^{2} d^{2} M_{0}(K)^{2} K^{2}+2 c^{2}\right] \sup _{0 \leq t \leq T}\left(\|V(t)\|_{H^{m+1}}^{2}+\left\|V_{t}(t)\right\|_{H^{m}}^{2}\right) \\
& +6 T^{2}\left[C^{2} d^{2} M_{0}(K)^{2}\left(K^{4}+K^{2}\right) \sup _{0 \leq t \leq T}\left(\|V(t)\|_{H^{m+1}}^{2}+\left\|V_{t}(t)\right\|_{H^{m}}^{2}\right)\right. \\
& \left.+4 c^{2} \sup _{0 \leq t \leq T}\left\|V_{t t}(t)\right\|_{H^{m-1}}^{2}\right] \\
\leq \quad & 18\left(T+T^{2}\right) e^{3 T}\left[C^{2} d^{2} M_{0}(K)^{2}\left(K^{4}+K^{2}\right)+6 c^{2}\right] \sup _{0 \leq t \leq T}\left[\|V(t)\|_{H^{m+1}}^{2}\right. \\
& \left.+\left\|V_{t}(t)\right\|_{H^{m}}^{2}+\left\|V_{t t}(t)\right\|_{H^{m-1}}^{2}\right] .
\end{aligned}
$$

If $K, T$ satisfy (3.15) and (3.16) respectively, and

$$
T \leq \min \left\{1,\left[72 e^{3} C^{2} d^{2} M_{0}(K)^{2}\left(K^{4}+K^{2}\right)+6 c^{2}\right]^{-1}\right\}
$$

thus, $A$ is strictly contractive.

Step 3. By the contraction mapping principle, we deduce that $A$ has a unique fixed point $w(x, t)(\in B(K, T))$ which is a solution to the problem (3.2)-(3.3). By virtue of integral estimates and the Gronwall inequality we know that problem (3.2)-(3.3) has at most a solution belonging to $Y\left(T^{\prime}\right)$ for each $T^{\prime}>0$, where $Y\left(T^{\prime}\right)=C\left(\left[0, T^{\prime}\right) ; H^{m+1}\right) \cap C^{1}\left(\left[0, T^{\prime}\right) ; H^{m}\right) \cap C^{2}\left(\left[0, T^{\prime}\right) ; H^{m-1}\right)$. Therefore, the Cauchy problem (3.2)-(3.3) admits a unique local solution $w(x, t)$ with

$$
w \in C\left(\left[0, T^{0}\right) ; H^{m+1}\right) \cap C^{1}\left(\left[0, T^{0}\right) ; H^{m}\right) \cap C^{2}\left(\left[0, T^{0}\right) ; H^{m-1}\right),
$$

where $\left[0, T^{0}\right)$ is the maximal existence interval, $T^{0}>0$.

Now we prove that if (3.6) holds, then $T_{0}=+\infty$.

In fact, assume that (3.6) holds and $T_{0}<+\infty$. For any $T^{\prime} \in\left[0, T_{0}\right)$, we consider the following Cauchy problem

$$
\begin{aligned}
& \widetilde{w}_{t t}-\triangle \widetilde{w}=k_{1} \sigma\left(P * \widetilde{w}_{x}\right)_{x}-P * \widetilde{w}+\widetilde{w} \\
& \widetilde{w}(x, 0)=w\left(x, T^{\prime}\right), \quad \widetilde{w}_{t}(x, 0)=w_{t}\left(x, T^{\prime}\right) .
\end{aligned}
$$


Observe that

$$
\|w(t)\|_{H^{m+1}}^{2}+\left\|w_{t}(t)\right\|_{H^{m}}^{2}+\left\|w_{t t}(t)\right\|_{H^{m-1}}^{2}
$$

is uniformly bounded about $T^{\prime} \in\left[0, T_{0}\right)$, and thus we choose $\widetilde{T} \in\left(0, T_{0}\right)$ such that for each $T^{\prime} \in\left[0, T_{0}\right)$, the Cauchy problem (3.24)-(3.25) has a unique solution $\widetilde{w} \in Y(\widetilde{T})$. The existence of such a $\widetilde{T}$ can be obtained by the contraction mapping principle. Particularly, (3.16) and (3.23) imply that $\widetilde{T}$ can be chosen independently of $T^{\prime} \in\left[0, T_{0}\right)$. Set $T^{\prime}=T_{0}-\widetilde{T} / 2$, Let $\widetilde{w}$ denote the corresponding solution to the Cauchy problem (3.24)-(3.25), and define $\hat{w}(x, t)$ by

$$
\hat{w}(x, t)= \begin{cases}w(x, t), & t \in\left[0, T^{\prime}\right], \\ \widetilde{w}\left(x, t-T^{\prime}\right), & t \in\left[T^{\prime}, T_{0}+\frac{\widetilde{T}}{2}\right] .\end{cases}
$$

By the construction of $\hat{w}(x, t), \hat{w}(x, t)$ is a solution to the Cauchy problem (3.2)(3.3) on $\left[0, T_{0}+\frac{\widetilde{T}}{2}\right]$, and by the local uniqueness, $\hat{w}$ extends $w$, which contradict the fact that the maximal time of existence is $\left[0, T_{0}\right)$. Hence, if (3.6) holds, then $T_{0}=+\infty$. Lemma 3.3 is proved.

Proof of Theorem 2.1. By Lemma 3.3, Lemma 3.1 and the equivalence relation between the Cauchy problem (1.8)-(1.9) and (3.2)-(3.3), we immediately obtain Theorem 2.1. Theorem 2.1 is proved.

\section{Existence and uniqueness of the global classical solution}

Proof of Theorem 2.2. Let $\phi_{i}(s)=\sigma_{i}(s)-\sigma_{i}(0)-k_{i} s$, where $k_{i}=\min \left\{0, C_{i}\right\}$, then $\phi_{i}(s)$ is a monotonically increasing function and $\phi_{i}(0)=0$, and hence

$$
\int_{0}^{s} \phi_{i}(\tau) d \tau \geq 0
$$

Rewrite the equation (1.8) as follows

$$
u_{t t}-\triangle u-\triangle u_{t t}+\triangle^{2} u-c \triangle u_{t}=d \sum_{i=1}^{N}\left[\frac{\partial}{\partial x_{i}} \phi_{i}\left(u_{x_{i}}\right)+k_{i} \frac{\partial^{2} u}{\partial x_{i}^{2}}\right] .
$$

Multiplying both sides of (4.1) by $u_{t}$ and integrating the resulting expression over $(-\infty,+\infty)$, adding $\left(u, u_{t}\right)$ to the both sides and using the Cauchy inequality we get

$$
\begin{aligned}
& \frac{d}{d t}\left(\|u(t)\|^{2}+\|\nabla u(t)\|^{2}+\left\|u_{t}(t)\right\|^{2}+\left\|\nabla u_{t}(t)\right\|^{2}+\|\triangle u(t)\|^{2}\right. \\
& \left.+2 d \sum_{i=1}^{N} \int_{R^{N}} \int_{0}^{u_{x_{i}}} \phi_{i}(s) d s d x\right)+2 c\left\|\nabla u_{t}(t)\right\|^{2} \\
\leq & d \sum_{i=1}^{N}\left|k_{i}\right|\left(\left\|u_{x_{i}}(t)\right\|^{2}+\left\|u_{x_{i} t}(t)\right\|^{2}\right)+\|u(t)\|^{2}+\left\|u_{t}(t)\right\|^{2} \\
\leq & d J\left(\|\nabla u(t)\|^{2}+\left\|\nabla u_{t}(t)\right\|^{2}\right)+\|u(t)\|^{2}+\left\|u_{t}(t)\right\|^{2},
\end{aligned}
$$

where $J=\max _{1 \leq i \leq N}\left\{\left|k_{i}\right|\right\}$. Making use of the Gronwall inequality we obtain

$$
\begin{aligned}
& \|u(t)\|^{2}+\|\nabla u(t)\|^{2}+\left\|u_{t}(t)\right\|^{2}+\left\|\nabla u_{t}(t)\right\|^{2} \\
& +\|\triangle u(t)\|^{2} \leq C_{1}(T), \quad t \in[0, T],
\end{aligned}
$$

here and in the sequel $C_{i}(T)(i=1,2,3,4)$ denote constants depending on $T$. 
Multiplying both sides of equation (1.8) by $\nabla^{2 m+2} u_{t}$, integrating the resulting expression over $(-\infty,+\infty)$ and integrating by parts we get

$$
\begin{gathered}
\frac{d}{d t}\left(\left\|\nabla^{m+1} u_{t}(t)\right\|^{2}+\left\|\nabla^{m+2} u(t)\right\|^{2}+\left\|\nabla^{m+2} u_{t}(t)\right\|^{2}+\left\|\nabla^{m+3} u(t)\right\|^{2}\right) \\
(4.4)=-2 d \sum_{i=1}^{N} \int_{R^{N}} \nabla^{m} \sigma_{i x_{i}}\left(u_{x_{i}}\right) \nabla^{m+2} u_{t} d x .
\end{gathered}
$$

From (4.3) and Sobolev imbedding theorem we know that $\nabla u \in L_{\infty}$, thus by using Lemma 2.1 and the Cauchy inequality we conclude that

$$
\begin{aligned}
& \left|\sum_{i=1}^{N} \int_{R^{N}} \nabla^{m} \sigma_{i x_{i}}\left(u_{x_{i}}\right) \nabla^{m+2} u_{t} d x\right| \\
\leq & M_{0}\left(\|\nabla u(t)\|_{\infty}\right)\left(\|\nabla u(t)\|+\left\|\nabla^{m+2} u(t)\right\|\right)\left\|\nabla^{m+2} u_{t}(t)\right\| .
\end{aligned}
$$

Substituting (4.5) into (4.4) we have

$$
\begin{aligned}
& \frac{d}{d t}\left(\left\|\nabla^{m+1} u_{t}(t)\right\|^{2}+\left\|\nabla^{m+2} u_{t}(t)\right\|^{2}+\left\|\nabla^{m+2} u(t)\right\|^{2}+\left\|\nabla^{m+3} u(t)\right\|^{2}\right) \\
\leq \quad & 2 d M_{1}\left(C_{1}(T)\right)\left[\left\|\nabla^{m+1} u_{t}(t)\right\|^{2}+\left\|\nabla^{m+2} u(t)\right\|^{2}\right. \\
& \left.+\left\|\nabla^{m+2} u_{t}(t)\right\|^{2}+\left\|\nabla^{m+3} u(t)\right\|^{2}\right],
\end{aligned}
$$

where $M_{1}\left(C_{1}(T)\right)$ are constants depending on $C_{1}(T)$. Applying the Growall inequality to (4.6) we get

(4.8) $\left\|\nabla^{m+1} u_{t}(t)\right\|^{2}+\left\|\nabla^{m+2} u_{t}(t)\right\|^{2}+\left\|\nabla^{m+3} u(t)\right\|^{2} \leq C_{2}(T), t \in[0, T]$.

Therefore, it follows from (4.3) and (4.7) that

$$
\|u(t)\|_{H^{m+3}}^{2}+\left\|u_{t}(t)\right\|_{H^{m+2}}^{2} \leq C_{3}(T), t \in[0, T)
$$

From equation (3.1) we obtain

$$
u_{t t}=P *\left[d \sum_{i=1}^{N} \frac{\partial}{\partial x_{i}} \sigma_{i}\left(u_{x_{i}}\right)+c u_{t}\right]+\Delta u-c u_{t} .
$$

By Lemma 3.1, Lemma 2.1 and (4.9) we get

$$
\begin{aligned}
& \left\|u_{t t}(t)\right\|_{H^{m+1}} \leq d M_{0}\left(C_{1}(T)\right)\|\nabla u(t)\|_{H^{m}} \\
& +\|\triangle u(t)\|_{H^{m+1}}+2 c\left\|u_{t}(t)\right\|_{H^{m+1}} .
\end{aligned}
$$

And hence by (4.8) we have

(4.12) $\|u(t)\|_{H^{m+3}}^{2}+\left\|u_{t}(t)\right\|_{H^{m+2}}^{2}+\left\|u_{t t}(t)\right\|_{H^{m+1}}^{2} \leq C_{4}(T), t \in[0, T)$,

$$
\sup _{0 \leq t<T}\left[\|u(t)\|_{H^{m+3}}^{2}+\left\|u_{t}(t)\right\|_{H^{m+2}}^{2}+\left\|u_{t t}(t)\right\|_{H^{m+1}}^{2}\right]<+\infty,
$$

by Theorem 2.1, we get $T=+\infty$, namely, the Cauchy problem (1.8)-(1.9) admits a unique global generalized solution

$$
u \in C\left([0, \infty) ; H^{m+3}\right) \cap C^{1}\left([0, \infty) ; H^{m+2}\right) \cap C^{2}\left([0, \infty) ; H^{m+1}\right) .
$$


Furthermore, by using the imbedding theorem $\left(m>1+\frac{N}{2}\right)$, we obtain that the Cauchy problem (1.8)-(1.9) admits a unique global classical solution

$$
u \in C\left([0, \infty) ; C^{4}\left(R^{N}\right)\right) \cap C^{1}\left([0, \infty) ; C^{3}\left(R^{N}\right)\right) \cap C^{2}\left([0, \infty) ; C^{2}\left(R^{N}\right)\right) .
$$

Theorem 2.2 is proved.

\section{Blow up of solutions for the Cauchy problem (1.8)-(1.9)}

Proof of Theorem 2.3. By the assumptions of Theorem 2.3 we know that the Cauchy problem (1.8)-(1.9) admits a unique local solution. If Cauchy problem (1.8)(1.9) admits a global generalized solution, then multiplying both sides of equation (1.8) by $u_{t}$ and integrating the resulting expression over $R^{N}$ we get

$$
\begin{aligned}
& \frac{d}{d t}\left(\left\|u_{t}(t)\right\|^{2}+\|\nabla u(t)\|^{2}+\left\|\nabla u_{t}(t)\right\|^{2}+\|\Delta u(t)\|^{2}\right. \\
& \left.+2 d \sum_{i=1}^{N} \int_{R^{N}} \int_{0}^{u_{x_{i}}} \sigma_{i}(s) d s d x\right)+2 c\left\|\nabla u_{t}(t)\right\|=0,
\end{aligned}
$$

integrating both sides over $(0, t)$, we obtain the energy identity,

$$
\begin{aligned}
E(t)= & \left\|u_{t}(t)\right\|^{2}+\|\nabla u(t)\|^{2}+\left\|\nabla u_{t}(t)\right\|^{2}+\|\triangle u(t)\|^{2} \\
& +2 d \sum_{i=1}^{N} \int_{R^{N}} \int_{0}^{u_{x_{i}}} \sigma_{i}(s) d s d x+2 c \int_{0}^{t}\left\|\nabla u_{t}(\tau)\right\| d \tau=E(0),
\end{aligned}
$$

where

$$
E(0)=\left\|u_{1}\right\|^{2}+\left\|\nabla u_{0}\right\|^{2}+\left\|\nabla u_{1}\right\|^{2}+\left\|\triangle u_{0}\right\|^{2}+2 d \sum_{i=1}^{N} \int_{R^{N}} \int_{0}^{u_{0 x_{i}}} \sigma_{i}(s) d s d x .
$$

Let

$$
F(t)=\|u(t)\|^{2}+\|\nabla u(t)\|^{2}+\beta(t+\eta)^{2},
$$

where $\beta$ and $\eta$ are nonnegative real numbers to be given later. Then

$$
F^{\prime}(t)=2\left[\left(u, u_{t}\right)+\left(\nabla u, \nabla u_{t}\right)+\beta(t+\eta)\right] .
$$

It follows from (5.3) and the Schwartz inequality that

$$
\begin{aligned}
F^{\prime}(t)^{2} & \leq 4\left[\|u(t)\|\left\|u_{t}(t)\right\|+\|\nabla u(t)\|\left\|\nabla u_{t}(t)\right\|+\sqrt{\beta} \sqrt{\beta}(t+\eta)\right]^{2} \\
& \leq 4 F(t)\left[\left\|u_{t}(t)\right\|^{2}+\left\|\nabla u_{t}(t)\right\|^{2}+\beta\right] .
\end{aligned}
$$

By (5.3) and equation (1.8) we have

$$
\begin{aligned}
F^{\prime \prime}(t)= & 2\left[\left\|u_{t}(t)\right\|^{2}+\left(u, u_{t t}\right)+\left\|\nabla u_{t}(t)\right\|^{2}+\left(\nabla u, \nabla u_{t t}\right)+\beta\right] \\
= & 2\left[\left\|u_{t}(t)\right\|^{2}-\|\nabla u(t)\|^{2}-c\left(\nabla u, \nabla u_{t}\right)-d \sum_{i=1}^{N}\left(\sigma_{i}\left(u_{x_{i}}\right), u_{x_{i}}\right)\right. \\
& \left.-\|\triangle u(t)\|^{2}+\left\|\nabla u_{t}(t)\right\|^{2}+\beta\right] .
\end{aligned}
$$


Hence by (5.4) and (5.5) we get

$$
\begin{aligned}
& F(t) F^{\prime \prime}(t)-(1+\gamma) F^{\prime}(t)^{2} \\
\geq & 2 F(t)\left\{\left[\left\|u_{t}(t)\right\|^{2}-\|\nabla u(t)\|^{2}-c\left(\nabla u, \nabla u_{t}\right)-d \sum_{i=1}^{N}\left(\sigma_{i}\left(u_{x_{i}}\right), u_{x_{i}}\right)\right.\right. \\
& \left.\left.-\|\triangle u(t)\|^{2}+\left\|\nabla u_{t}(t)\right\|^{2}+\beta\right]-2(1+\gamma)\left[\left\|u_{t}(t)\right\|^{2}+\left\|\nabla u_{t}(t)\right\|^{2}+\beta\right]\right\} \\
(5.6)= & 2 F(t) F_{1}(t),
\end{aligned}
$$

where

$$
\begin{aligned}
F_{1}(t)= & -(1+2 \gamma)\left[\left\|u_{t}(t)\right\|^{2}+\left\|\nabla u_{t}(t)\right\|^{2}+\beta\right]-d \sum_{i=1}^{N}\left(\sigma_{i}\left(u_{x_{i}}\right), u_{x_{i}}\right) \\
& -\left\|\nabla u_{t}(t)\right\|^{2}-\|\triangle u(t)\|^{2}-c\left(\nabla u, \nabla u_{t}\right)
\end{aligned}
$$

By (5.7) and equation (1.8) we obtain

$$
\begin{aligned}
F_{1}^{\prime}(t)= & -2(1+2 \gamma)\left[\left(u_{t}, u_{t t}\right)-\left(u_{t}, \triangle u_{t t}\right)\right]-\frac{d}{d t}\left[d \sum_{i=1}^{N}\left(\sigma_{i}\left(u_{x_{i}}\right), u_{x_{i}}\right)+\|\Delta u(t)\|^{2}\right. \\
& \left.+\|\nabla u(t)\|^{2}+c\left(\nabla u, \nabla u_{t}\right)\right] \\
= & (1+2 \gamma)\left[\frac{d}{d t}\left(\|\nabla u(t)\|^{2}+\|\Delta u(t)\|^{2}+2 d \sum_{i=1}^{N} \int_{R^{N}} \int_{0}^{u_{x_{i}}} \sigma_{i}(s) d s d x\right)\right. \\
& \left.+2 c\left\|\nabla u_{t}(t)\right\|^{2}\right]-\frac{d}{d t}\left[d \sum_{i=1}^{N}\left(\sigma_{i}\left(u_{x_{i}}\right), u_{x_{i}}\right)+\|\Delta u(t)\|^{2}\right. \\
& \left.+\|\nabla u(t)\|^{2}+c\left(\nabla u, \nabla u_{t}\right)\right] .
\end{aligned}
$$

Observe that

$$
\begin{aligned}
c\left(\nabla u, \nabla u_{t}\right) & \leq c^{2}\|\nabla u(t)\|^{2}+\left\|\nabla u_{t}(t)\right\|^{2} \\
& \leq c^{2} \sum_{i=1}^{N} \int_{R^{N}} u_{x_{i}}^{2} d x+E(0)-d \sum_{i=1}^{N} \int_{R^{N}} \int_{0}^{u_{x_{i}}} \sigma_{i}(s) d s d x
\end{aligned}
$$


from (5.8) and condition (2) of Theorem 2.3 we have

$$
\begin{aligned}
& F_{1}(t)=(1+2 \gamma)\left[\|\nabla u(t)\|^{2}+\|\Delta u(t)\|^{2}\right. \\
& \left.+2 d \sum_{i=1}^{N} \int_{R^{N}} \int_{0}^{u_{x_{i}}} \sigma_{i}(s) d s d x+2 c \int_{0}^{t}\left\|\nabla u_{t}(\tau)\right\|^{2} d \tau\right] \\
& -\left[d \sum_{i=1}^{N}\left(\sigma_{i}\left(u_{x_{i}}\right), u_{x_{i}}\right)+\|\Delta u(t)\|^{2}+\|\nabla u(t)\|^{2}+c\left(\nabla u, \nabla u_{t}\right)\right] \\
& +F_{1}(0)-(1+2 \gamma)\left[\left\|\nabla u_{0}\right\|^{2}+\left\|\Delta u_{0}\right\|^{2}\right. \\
& \left.+2 d \sum_{i=1}^{N} \int_{R^{N}} \int_{0}^{u_{x_{i}}} \sigma_{i}(s) d s d x\right] \\
& +\left[d \sum_{i=1}^{N}\left(\sigma_{i}\left(u_{0 x_{i}}\right), u_{0 x_{i}}\right)+\left\|\triangle u_{0}\right\|^{2}+\left\|\nabla u_{0}\right\|^{2}+c\left(\nabla u_{0}, \nabla u_{1}\right)\right] \\
& \geq(1+2 \gamma)\left[\left\|u_{1}\right\|^{2}+\left\|\nabla u_{1}\right\|^{2}+\left\|\nabla u_{0}\right\|^{2}+\left\|\Delta u_{0}\right\|^{2}\right. \\
& \left.+2 d \sum_{i=1}^{N} \int_{R^{N}} \Gamma_{i}\left(u_{0 x_{i}}\right) d x+\beta\right] \\
& +\sum_{i=1}^{N} \int_{R^{N}}\left[(3+4 \gamma) d \Gamma_{i}\left(u_{x_{i}}\right)-d \sigma_{i}\left(u_{x_{i}}\right) u_{x_{i}}-c^{2} u_{x_{i}}^{2}\right] d x-E(0) \\
& \quad-2(1+\gamma) E(0)-(1+2 \gamma) \beta .
\end{aligned}
$$

(1) If $E(0)<0$, choosing $\beta=-\frac{2(1+\gamma)}{1+2 \gamma} E(0)$, then

$$
F(t) F^{\prime \prime}(t)-(1+\gamma) F^{\prime}(t)^{2} \geq 0,
$$

and $F(0)>0, F^{\prime}(0)>0$ if $\eta$ is large enough, by Lemma 2.3 , we obtain that $F(t) \rightarrow+\infty$ as $t \rightarrow t_{1} \leq t_{2}=F(0) / \gamma F^{\prime}(0)=\left(\left\|u_{0}\right\|^{2}+\left\|\nabla u_{0}\right\|^{2}+\beta \eta^{2}\right) / 2 \gamma\left[\left(u_{0}, u_{1}\right)+\right.$ $\left.\left.\left(\nabla u_{0}, \nabla u_{1}\right)+\beta \eta\right)\right]$, and hence $\|u(t)\|^{2}+\|\nabla u(t)\|^{2} \rightarrow+\infty$ as $t \rightarrow t_{1}$.

(2) If $E(0)=0$, choosing $\beta=0$, then

$$
F(t) F^{\prime \prime}(t)-(1+\gamma) F^{\prime}(t)^{2} \geq 0,
$$

and $F^{\prime}(0)>0$ by assumption $\left(u_{0}, u_{1}\right)+\left(\nabla u_{0}, \nabla u_{1}\right)>0$, by lemma 2.3 , we obtain that $F(t) \rightarrow+\infty$ as $t \rightarrow t_{1} \leq t_{2}=F(0) / \gamma F^{\prime}(0)=\left(\left\|u_{0}\right\|^{2}+\left\|\nabla u_{0}\right\|^{2}\right) / 2 \gamma\left[\left(u_{0}, u_{1}\right)+\right.$ $\left.\left(\nabla u_{0}, \nabla u_{1}\right)\right]$, and hence $\|u(t)\|^{2}+\|\nabla u(t)\|^{2} \rightarrow+\infty$ as $t \rightarrow t_{1}$.

(3) If $E(0)>0$, choosing $\beta=0$, from (5.6) and (5.9) we have

$$
F(t) F^{\prime \prime}(t)-(1+\gamma) F^{\prime}(t)^{2} \geq-4(1+\gamma) E(0) F(t) .
$$

Let $H(t)=F^{-\gamma}(t)$, then

$$
\begin{aligned}
H^{\prime}(t) & =-\gamma F^{-\gamma-1}(t) F^{\prime}(t) \\
H^{\prime \prime}(t) & =-\gamma(-\gamma-1) F^{-\gamma-2}(t) F^{\prime}(t)^{2}-\gamma F^{-\gamma-1}(t) F^{\prime \prime}(t) \\
& =-\gamma F^{-\gamma-2}(t)\left[F(t) F^{\prime \prime}(t)-(1+\gamma) F^{\prime}(t)^{2}\right] \\
& \leq 4(1+\gamma) \gamma E(0) F^{-\gamma-1}(t) .
\end{aligned}
$$


By the assumption (iii) of theorem 2.3 we know that

$$
H^{\prime}(0)=-\gamma F^{-\gamma-1}(0) F^{\prime}(0)<0 .
$$

Let

$$
T=\sup \left\{\tau \mid H^{\prime}(\tau)<0, \tau \in[0, t)\right\},
$$

by the continuity of $H^{\prime}(t)$, we get $T>0$. Multiplying (5.11) by $2 H^{\prime}(t)$ yields

$$
\begin{aligned}
\frac{d}{d t} H^{\prime}(t)^{2} & \geq-8 \gamma^{2}(1+\gamma) E(0) F^{-2 \gamma-2}(t) F^{\prime}(t) \\
& =\frac{8 \gamma^{2}(1+\gamma)}{1+2 \gamma} E(0)\left[F^{-2 \gamma-1}(t)\right]^{\prime}, t \in[0, T)
\end{aligned}
$$

Integrating both sides of (5.13) over $[0, T)$ we obtain

$$
\begin{aligned}
& H^{\prime}(t)^{2} \geq H^{\prime}(0)^{2}+ \\
& \frac{8 \gamma^{2}(1+\gamma)}{1+2 \gamma} E(0)\left[F^{-2 \gamma-1}(t)-F^{-2 \gamma-1}(0)\right], t \in[0, T) .
\end{aligned}
$$

It follows from the condition (iii) of theorem 2.3 that

$$
H^{\prime}(0)^{2}-\frac{8 \gamma^{2}(1+\gamma)}{1+2 \gamma} E(0) F^{-2 \gamma-1}(0)>0
$$

So by the continuity of $H^{\prime}(t),(5.14)$ and (5.15) we conclude that

$$
H^{\prime}(t) \leq-\left[H^{\prime}(0)^{2}-\frac{8 \gamma^{2}(1+\gamma)}{1+2 \gamma} E(0) F^{-2 \gamma-1}(0)\right]^{1 / 2}, t \in[0, T),
$$

by the definition of $T,(5.16)$ holds for all $t \geq 0$. Therefore

$$
H(t) \leq H(0)-\left[H^{\prime}(0)^{2}-\frac{8 \gamma^{2}(1+\gamma)}{1+2 \gamma} E(0) F^{-2 \gamma-1}(0)\right]^{1 / 2} t, t>0
$$

and hence $H\left(T_{1}\right)=0$ for some $T_{1}$, and $0<T_{1} \leq T_{0}$, with

$$
T_{0}=H(0)\left[H^{\prime}(0)^{2}-\frac{8 \gamma^{2}(1+\gamma)}{1+2 \gamma} E(0) F^{-2 \gamma-1}(0)\right]^{-1 / 2} .
$$

So $F(t) \rightarrow+\infty$ as $t \rightarrow T_{1} \leq T_{0}$, namely, $\|u(t)\|^{2}+\left\|u_{x}(t)\right\|^{2} \rightarrow \infty$ as $t \rightarrow T_{1}$.

Therefore, if the conditions of Theorem 2.3 holds, then $\|u(t)\|^{2}+\left\|u_{x}(t)\right\|^{2}$ becomes infinite at a finite time. Theorem 2.3 is proved.

An example. We take $N=1, \sigma_{i}(s)=a_{0} s^{p}+s$, where $a_{0} \neq 0$ is a constant and $p$ is a positive integer. And take initial data

$$
u_{0}(x)=u_{1}(x)= \begin{cases}k_{0} \frac{d}{d x} e^{\frac{1}{x^{2}-1}}, & |x|<1, \\ 0, & |x| \geq 1\end{cases}
$$

where $k_{0} \neq 0$ is a constant. Obviously $u_{0}, u_{1} \in C_{0}^{\infty}\left(R^{1}\right)$.

(1) When $a_{0}>0$ and $p$ is an odd number, a simple verification shows that all assumptions of the Theorem 2.2 are satisfied, then by Theorem 2.2 we obtain that the Cauchy problem (1.8)-(1.9) admits a unique global classical solution $u(x, t)$ with

$$
u \in C\left([0, \infty) ; C^{4}\right) \cap C^{1}\left([0, \infty) ; C^{3}\right) \cap C^{2}\left([0, \infty) ; C^{2}\right)
$$


(2) When $a_{0}>0$ and $p>2$ is an even number, $c=d=1$, a simple calculation shows that condition (2) of Theorem 2.3 is satisfied, with $\Gamma_{i}(s)=a_{0} s^{p+1} /(p+1)+$ $s^{2} / 2, \gamma=(p-2) / 4$. Taking $\left|k_{0}\right|$ suitable large such that

$$
\frac{2 d a_{0}}{p+1} k_{0}^{P+1} \int_{|x|<1}\left(\frac{d^{2}}{d x^{2}} e^{\frac{1}{x^{2}-1}}\right)^{p+1} d x<0
$$

and

$$
\begin{aligned}
E(0)= & \left\|u_{1}\right\|^{2}+\left\|u_{0 x}\right\|^{2}+\left\|u_{1 x}\right\|^{2}+\left\|u_{0 x x}\right\|^{2}+2 d \int_{R^{1}} \int_{0}^{u_{0 x}} \sigma(s) d s d x \\
\leq & k_{0}^{2}\left[\int_{|x|<1}\left(\frac{d}{d x} e^{\frac{1}{x^{2}-1}}\right)^{2} d x+(2+d) \int_{|x|<1}\left(\frac{d^{2}}{d x^{2}} e^{\frac{1}{x^{2}-1}}\right)^{2} d x\right. \\
& \left.+\int_{|x|<1}\left(\frac{d^{3}}{d x^{3}} e^{\frac{1}{x^{2}-1}}\right)^{2} d x\right] \\
& +\frac{2 d a_{0}}{p+1} k_{0}^{P+1} \int_{|x|<1}\left(\frac{d^{2}}{d x^{2}} e^{\frac{1}{x^{2}-1}}\right)^{p+1} d x<0 .
\end{aligned}
$$

Therefore, by Theorem 2.3, the solution of the Cauchy problem (1.8)-(1.9) must blow up at a finite time.

(3) When $a_{0}<0$ and $p$ is an odd number, $c=d=1$, a simple computation shows that condition (2) of Theorem 2.3 is satisfied, with $\Gamma(s)=a_{0} s^{p+1} /(p+1)+$ $s^{2} / 2, \gamma=(p-2) / 4$. Taking $k_{0}>0$ suitable large such that $E(0)<0$, then the solution of the Cauchy problem (1.8)-(1.9) must blow up at a finite time.

\section{References}

[1] J. Boussinesq, Thé orie des ondes et des remous qui se propagent le long $\mathrm{d}^{\prime}$ un canal rectangulaire horizontal en communiquant an liquide contenu dans ce canal des vitesses sensiblement pareilles de la surface au fond, J. Math. Pures Appl., 17(2) (1872), 55-108.

[2] V. Varlamov, Existence and uniqueness of solutions to the Cauchy problem for the damped Boussinesq equation, Math. Meth. Appl. Sci. 19 (1996), 639-649.

[3] J. L. Bona, R. A. Smith, A model for the two-way propagation of water waves in a channel, Math. Proc. Cambridge Philos. Soc. 79 (1976), 167-182.

[4] J. L. Bona, R. L. Sachs, Global existence of smooth solutions and stability of solitary waves for a generalized Boussinesq equation, Commun. Math. Phys. 118 (1988), 15-19.

[5] F. -L. Liu and D. Russel, Solutions of the Boussinesq equation on a peridic domain, J. Math. Anal. Appl. 192 (1995), 194-219.

[6] J. Defrutos, T. Ortega, J. M. Sanz-Serna, Pseudospectral method for the "good" Boussinesq equation, Math. Comp. 57 (1991), 109-122.

[7] Chen Guowang, Yang Zhijian, Existence and non-existence of global solutions for a class of non-linear wave equation, Math. Meth. Appl. Sci. 23 (2000), 615-631.

[8] T. Kano and T. Nishida, A mathematical justification for Korteweg-de Vries equation and Boussinesq equation of water surface waves. Osaka J. Math., 23(1986), 389-413.

[9] M. E. Taylor, Partial Diffarantial Equations III, Nonlinear Equations, Spring-Verlag, New York (1996).

[10] F. Linares, Global existence of small solutions for a generalized Boussinesq equation, J. Differential Equations 106 (1993), 257-293.

[11] M. J. Ablowitz and P. A. Clarkson, Solitons, nonlinear evolution equations and inverse scattering, London Math. Soc. Lecture Note Series, $\sharp 149$, Cambridge University Press, Cambridge (1991), $516 \mathrm{pp}$

[12] P. Naumkin and I. Shismare, Nonlinear nonlocal equations in the theorem of waves, Transl. of Math. Monographs, Vol.133, Amer. Math. Soc. Providence, Rhode Island (1994).

[13] A. K. Pani, H. Sarange, Finite element Galerkin method for the "good" Boussinesq equation, Nonlinear Anal. TMA 29 (1997), 937-956. 
[14] R. L. Sachs, On the blow-up of certain solutions of the "good" Boussinesq equation, Appl. Anal. 36 (1990), 145-152.

[15] P. Biler, Decay of solutions of semilinear strongly damped generalized wave equation, Math. Meth. Appl. Sci., 14 (6) (1991), 427-444.

[16] V. K. Kalantarov and O. A. Ladyzhenskaya, The occurrence of collapse for quasilinear equations of parabolic and hyperbolic types, J. Soviet Math., 10 (1978), 53-70.

[17] Li Ta-Tsien and Yuen-mei Chen, "Nonlinear Evolution Equations," Science Press, Beijing (1989) (in Chinese).

[18] R. A. Admas, Sobolev Space, Acad. Press, N. Y.(1975).

[19] E. M. Stein, Singular Integrals and Differentiability Properties of Function, Princeton Univ. Press, Princeton, NJ (1970).

Department of Mathematics, Zhongyuan University of Technology, Zhengzhou 450007, P. R. CHINA

E-mail address: cmsongh@163.com

Department of Mathematics, Zhengzhou University, Zhengzhou 450052, P. R. China

E-mail address: yzjzzut@tom.com 